\title{
THE INTERNET OF THINGS (IOT) A SURVEY
}

\author{
Rouhollah Nabati \\ Master of Software Engineering, Hamedan Branch, Islamic Azad University, Hamedan, Iran \\ rnabati@gmail.com \\ Samira Taheri \\ Master of Software Engineering, Hamedan Branch, Islamic Azad University, Hamedan, Iran \\ taheri.mst0@gmail.com
}

\begin{abstract}
The present study aims to review the latest status of Internet of Things. With the advent of Internet of Things, a new type of relationship emerged between objects and human as well as objects and objects. This technology was manifested by combining different technologies of communication and information and has created new dimensions in the world of communications. We can clearly perceive that the development of this technology requires serious efforts in various areas of knowledge including Communications and Information Technology (ICT), Informatics, Electronics and Social Engineering. In present paper, we attempted to investigate and compare different views about the Internet of Things in scientific and academic communities. We also study different technologies such as RFID -based systems and wireless sensor networks which are enabling technologies. Afterwards different applications of Internet of Things in everyday life and industry and finally the issues which demand further research and development will be reviewed.
\end{abstract}

Keywords: Internet of Things, WSN, RFID, wireless sensor networks, examination

\section{INTRODUCTION}

A new era of wireless communication technology called Internet of Things (IoT) has been emerged in recent years. The Internet of Things was introduced by Kevin Ashton [4] for the first time in a conference in 1999. Then many studies conducted on this subject in the scientific and academic communities. In fact, the Internet of Things is a new phenomenon in the field of wireless communications which is expanding and will outstrip it rivals in near future. The main idea is based on the assumption that different electrical equipment surrounding our life such as sensors, tags, mobile phones etc. communicate with each other using Radio Frequency Identification (RFID), form a small network and send and receive information with their neighbors [6]. The realization of IoT idea requires the establishment of relations between Radio Frequency Identification (RFID) systems (tracking, addressing), Wireless Sensor Networks (WSN) (collection of distributed data, data transmission and processing), Smart Technologies (Using knowledge to solve specific problems that usually insist on Artificial Intelligence such as Machine - machine communication systems and smart signal processing) and Nanotechnology (Production of Nano-sized devices usually 0.1 to $100 \mathrm{~nm}$ ). This technology will have significant impacts on human life such as working and personal affairs in coming future. Automation, management of industrial plants, business and management, smart transportation and health are instances of areas which will be completely changed by LoT [7].

With regard to the aforesaid cases, it is not surprising that this technology has been put in the list of six disruptive technologies of social security by National Intelligence Council and National Security Council of the United States of America [12] which directly affects the national security of America. According to NIC predictions, many of the everyday life objects such as food packages, clothing, beverage bottles, locations, home furniture, documents, paper, home appliances, television, etc. will be connected to 
internet up to 2025. The emergence of this technology is actually a combination of human needs with technology, similar to the existing Internet that will bring about enormous opportunities for scientific and economic development. Along with countless opportunities for development, the security threats arising from such development cannot be denied. Actually the connection of surrounding devices to Internet will make them security threats in a way that the security threats will be far more extensive than the current Internet security risks. Before the idea of IoT is widely accepted, several controversial issues should be studied so that optimal solutions are found and IoT implementation is achieved. The main noteworthy issue is the creation of intelligent devices which operate independently, have the ability to adapt, communicate and exchange data with their neighbors and at the same time protect the information and privacy with absolute guaranteed. Besides the idea of IoT with regard to its exclusive characteristics creates new problems in networking concepts. In fact the devices which realize the idea of IoT are recognized with properties such as low rate in both energy consumption and processing. Therefore, the proposed approaches require especial attention to resource efficiency along with scalability problems. Now several organizations and research centers endeavored to develop the solutions ideal for IoT infrastructures. But given that the IoT is just at the beginning of development path in all areas of life, a universally acceptable architecture is not established. At the present time, two EPC Global and UID methods are amongst the architectures mostly accepted by different research centers.

\section{EPCGlobal}

EPG Global is an active institution in the field of information technology which is established to achieve the global standard of Electronic Product Code (EPC). This institution is established with joint investment of GSI (formerly known as EAN International) and GSI US (formerly known as Uniform Code Council). Its mission is to develop industry standards for the EPC using RFID radio technology. The main idea of EPC Global is designing a system which is able to connect the objects using RFID frequencies based on Internet architecture [2]. In this architecture, each object obtains a unique electronic product code (EPC) as IP. It is managed by RFID management system (Aguirre, 2007).

The EPCGlobal (Figure 1) is composed of three exchange, capture and identify layers. Each layer consists of several parts which define different standards for that layer.

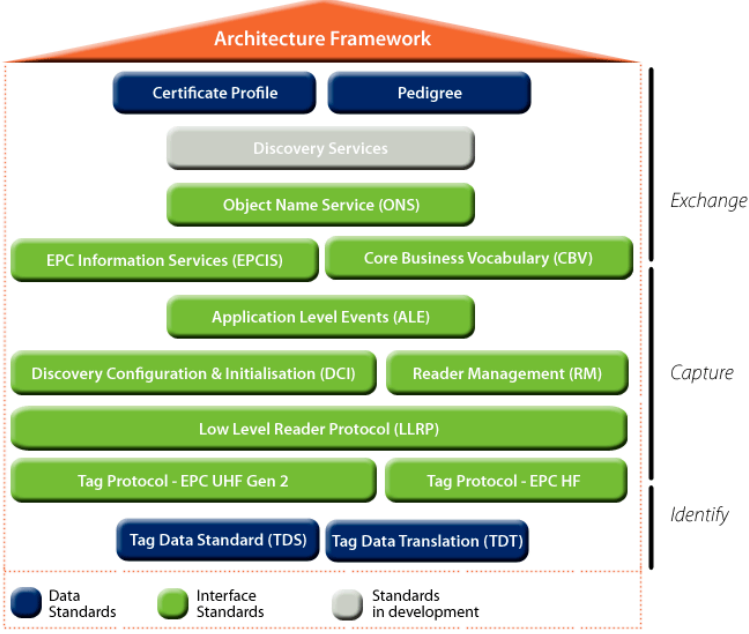

Figure (1): EPC Global

Another widely accepted architecture is UID which is the product of a Japanese institution managed by Mr. Sakamaro in Tokyo. UID is the abbreviated from of Unique / Ubiquitous / Universal Identification. This architecture tries to realize the idea of pervasive computing using a middle ware architecture. Pervasive computing is an emerging technology that aims to achieve a better lifestyle. In UID architecture, the entire surrounding devices (which are called things in terminology) including walls, furniture, roofs, houses, home electronics, etc. enter to the cycle of pervasive computing by installation of 
components to be autonomously controlled. Then these objects and entities can communicate with each other and exchange information [3].

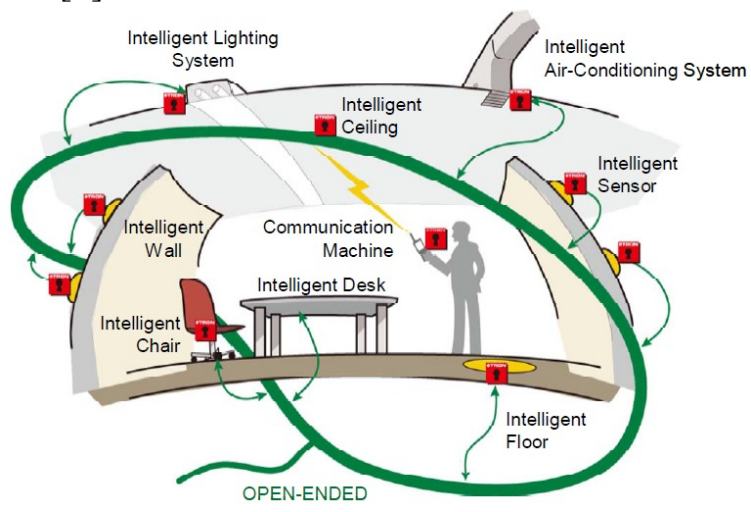

Figure (2): Devises installed inside home in UID

This technology is not restricted to internal equipment (Figure 2) and could be expanded out of buildings and homes (figure 3) such as panels and traffic signs, urban management equipment, pharmacies, libraries, places, etc. To identify each object, a unique identification code as ucode (Ubiquitous Code) is assigned.

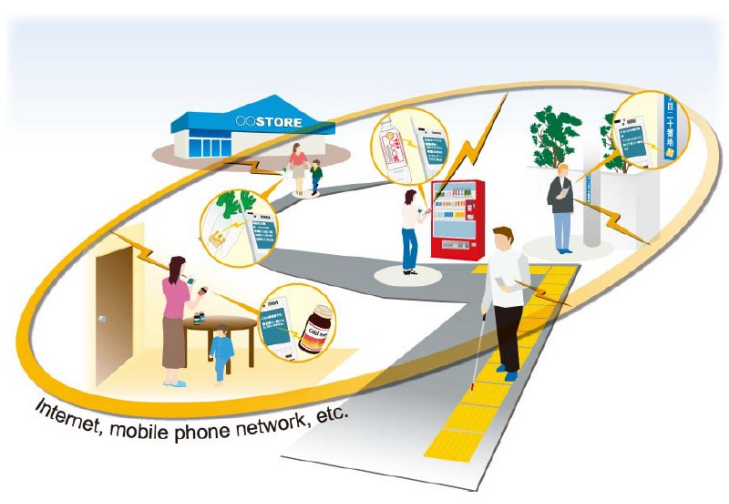

Figure (3): Relation between external objects in UID

\section{Mechanism and Main Components of UID Architecture}

\section{1-Ucode Tags}

Tags are electronic labels which store the identification codes of objects.

2-UC or Ubiquitous Communicator

UC is actually a terminal device used to read the tag data.

3- Ucode Relation Database

The database manages the data of objects in distributed form.

4- Ucode Information Server

In this architecture, the servers manage the data displayed by UC and required services.

There are abundant research and industry centers for the development of Internet of Things in many countries. We tried to investigate the latest status of objects in this paper.

In second section, different point of views on internet of Things proposed by industrial and academic communities are studied. In third section, technologies based on Internet of Things will be introduced. The different uses of Internet of Things in the areas of transportation, medicine, smart environment and social contexts are discussed in fourth section. The fifth section is allocated to open subjects for further researches. The sixth section includes discussion and conclusion of interpreted subjects. 


\section{Different Point of Views on Internet of Things}

Internet of Things has been studied from different aspects in recent years and developed in a variety of applications and different directions. Figure 2 demonstrates various attitudes on Internet of Things. Internet of Things is described as things-oriented in some texts, as Internet-oriented by scientific communities and semantic-oriented by some others.

\section{Things-oriented Point of View}

In Internet of Things, all surrounding equipment and objects are connected to Internet using tags. In this point of view, everything is regarded as an object. The RFID and NFC are amongst these properties. The WISP project by INTEL Company is one of the commercial projects produced on the basis of this point of view. IN this point of view, only the objects are connected to each other.

\section{Internet-Oriented Point of View}

In this point of view, the objects connected to each other by wireless technologies make an Internet of objects. In this way an Internet of Things could be found. IPSO or IP for smart things is the result of research teams which consider the Internet of Things from this point of view.

\section{Semantic-Oriented Point of View}

The sematic-oriented technology is the basis for this point of view. In sematic-oriented point of view, the objects are semantically researchable.

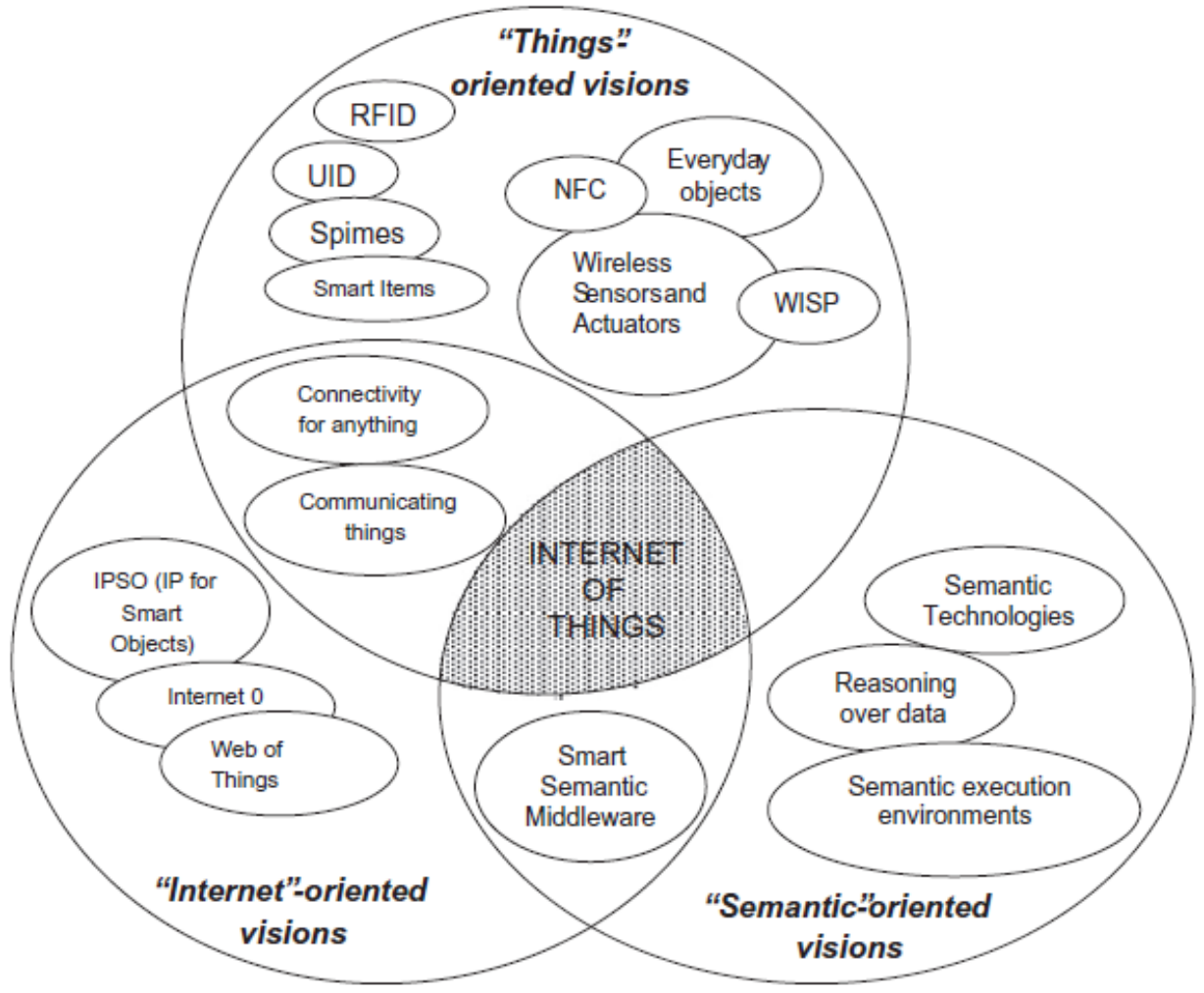

Figure (4): Internet of Things from Different Point of View

\section{Enabling Technologies}

The realization of Internet of things requires the combination and cooperation between different communication technologies. In this section, we will introduce several technologies which could play vital role in the process of realizing the idea of Internet of Things. The noteworthy point is that our purpose was not to render a comprehensive study of the details in these technologies, but we tried to demonstrate a general portrait of their roles in Internet of Things.

\section{Sensor Technologies and Sensor Communication Networks}


The idea of "Anytime, Anywhere, Any Media" has been the progressing vision for development of sensor technologies. The radio communication technology plays a major role in this vision [10]. Today the production of radio devices is approximately 1 to 1 . It means that for every human being on this planet there is nearly a device that works with radio frequency. However the reduction of radio devices in terms of size, weight or even energy consumption which are seriously popular will increase this ratio to ten times. This means that in near future there will be dozens of radio sensor devices for every human. With ever dwindling of these devises in size, it is possible to place them into every things or objects and so make the Internet of Things.

RFID will be the main part of these technologies which includes several signal reader devises (FRID reader) and abundant number of RFID tags. As previously mentioned, the object data will be recorded in tags. The RFID reader devices come near to object, read the information of these tags and conduct the respective process. Besides, the RFID technology make the immediate supervision on objects possible. In other words, by installing the tags and collecting the data by RFID readers, we could be informed of last place and position of respective object. IN physical term, tag is a very small chip attached on an object or placed into it which restore the electronic information. The tag is connected to a small antenna which sends and receives information by radio frequency. Hitachi Company has produced the smallest tag in $0.15^{*} 0.4 * 0.4 \mathrm{~mm}$ size. The RFID tags are usually inactive. In other words, they do not carry power supply of energy production and provide their energy needs through signals sent by RFID reader devices. In fact, the signals sent by RFID reader devices induce an electric current to tag antenna and provide the energy required by tags for transmission of information. The frequency taken by a RFID reader device is often received and processed by the same device. Since the energy induced to antenna is very slight, the tags existing in the range of that radio frequency or in few meter distance are able to receive them.

Sending and receiving the signals occur in different ranges from low frequency (LF) $124 \mathrm{kHz}$ to $135 \mathrm{kHz}$ and even Ultra High Frequencies (UHF) from860 to $960 \mathrm{MHz}$, However some of the tags provide their energy supply via batteries. In this section, we completely separate the two concepts of inactive and semiactive. IN semi-active RFID, the essential energy to receive signals are provided by microchips, but in active RFID, the energy transmission occurs by battery energy. So the frequency range of active RFID is much higher than inactive and semi-active RFIDs.

Sensor networks will also play a vital role to achieve the idea of the Internet of Things. In fact the sensor networks create close communications with RFID technology and are helpful in accurate tracking of the status of objects such as temperature, location and movements of objects. The extensive uses of sensor networks include environmental monitoring, e- health, intelligent transport systems, military systems, industrial environments, etc. The sensor networks include definite number of communication nodes (may be in large number) which communicate with each other in the form of a multi-hop communication network. In these networks, the nodes often announce their results to other nodes (often one node) named Sink.

In recent years, many researches have been implemented by scientists on sensor networks. These researches have been on all layers of communication protocols. Among the characteristics of this research inspired by the nature of sensor networks we can point to optimal energy efficiency, breadth, reliability and robustness. The optimal energy consumption should be noticed due to the fact that the sensors are located in areas which have limited access to energy sources and it is difficult for them to have access to energy supply. Expansion or in more precise term extensibility was regarded because of the reason that number of nodes in sensor networks could be very large and even in wide geographical distribution. Reliability also bears significant importance because these nodes should transmit vital and emergency information in some cases. Finally the resistance of sensors is vital because they may fail due to several factors. 
Nowadays, most of the sensor networks are created based on the IEEE 802.15.4 standard which define the physical layer and MAC layer (medium access layer) for communications with low energy consumption and data transmission rate in Wireless Personal Area Networks (WPAN) systems. The IEEE 802.15.4 has not defined other properties and standards of upper layers for the communication between one node and upper layers due to existing problems [1]. Some of these problems are as follows:

- Sensor networks usually include large number of sensors which make the task impossible due to the number of IP existing in version 4.

- According to IEEE 802.15.4 standard, the maximum packet size of physical layer is 127 bytes and the maximum packet size in MAC layer is 102 bytes which could be less in terms of encryption algorithms of data link layers. These sizes are very small compared to the size of the IP packets.

- In many cases of sensor networks, the nodes spend most of their time in sleep mode to save energy. They cannot make communication with network during this time which is very abnormal for IP networks.

The combination of sensor network technologies with inactive RFID tags will create specific applications in the area of E-health. In recent years, many solutions have been proposed in this area. For instance the WISP was introduced by laboratory of INTEL Company to develop the inactive RFID technology and sensor networks. The WISP has used the implemented RFID technology and provided the energy required by tags through signals sent by RFID readers. In this case the tags could measure certain environmental conditions such as temperature, brightness level, pressure and liquid level. By combining RFID systems and RFID sensor networks, sensor networks could be established [8] which include small sensor chips and processing RFID and RFID reader chips. The RFID readers are actually the network sinks which receive the data sent by nodes (tags) and provide the energy required by network. Table 1 compares the different technologies of RFID systems, WSN sensor network and RFID sensor networks. The advantages of each are as follows:

- The RFID systems are produced in small size and at low expense. However their lifetime is not restricted by battery (due to lack of battery) and work for a very long time.

- The wireless sensor networks possess higher frequency range and so do not require a reader node such as RFID reader. Because the communications in this networks are peer to peer and asymmetric.

- The RFID wireless sensor networks have the ability to implement the sensor operations and communications in inactive systems.

\section{Middle Wares}

The middle ware is a layer or combination of several software sub layers placed between technology and practical program. The middle ware task is to hide the details of technology which the user or program producer do not need to have direct relation with it. The middle ware has been greatly noticed in recent years due to its ability to simplify the development of applications and new services and it importance is increasing day by day. In fact it breaks off the relation between programmer and the details of lower layers or hide the details.

As occurred in other section, the middle ware usually follow the Software Oriented Architecture (SOA) approach. Using Software Oriented Architecture, a complex system could be divided into smaller parts which are actually parts of a very large system and were defined accurately. The categorization could present a horizontal view from an organizational system which is helpful for analysis. Besides, the Software Oriented Architecture provides the ground for reuse of hardware and software in the design process. In most researches, the advantages of Software Oriented Architecture approach in the Internet of Things has been proven. In case that an officially accepted and applied layer program confronted a problem and set aside for some reasons, the solutions proposed based on that approach will have the same problems. 
These procedures led to presentation of a general definition of middle ware which is demonstrated in Figure 5. This definition tries to cover the entire properties and results of previous researches on Internet of Things.

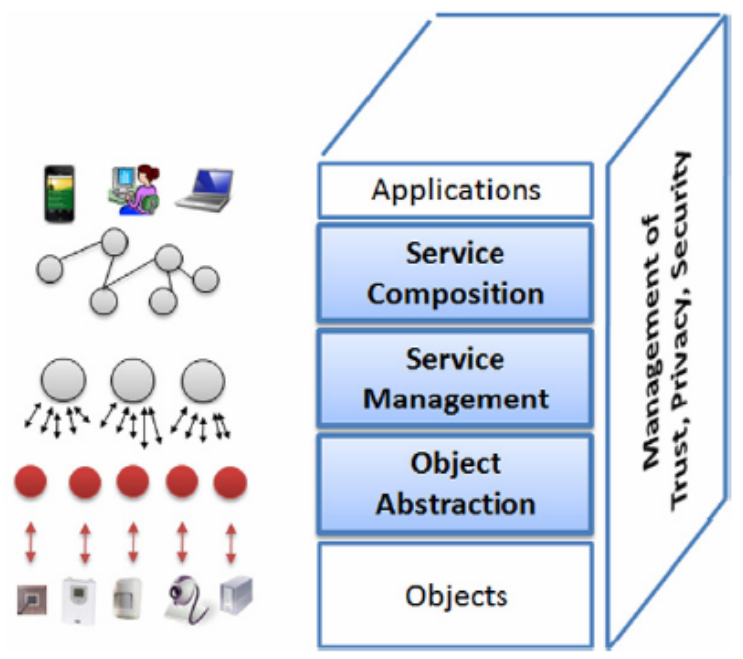

Figure (5): Software Oriented Architecture

Middle Ware Architecture include the following sub layers [5]:

- Middle ware uses

- Service combination layer

- Service management layer

- Object abstraction layer

- Object layer

Table 1: Comparison between RFID based systems, wireless sensor networks and RFID sensor networks

\begin{tabular}{lllllllll}
\hline & Processing & Sensing & Communication & Range $(\mathrm{m})$ & Power & Lifetime & Size & Standard \\
\hline RFID & No & No & Asymmetric & 10 & Harvested & Indefinite & Very small & ISO18000 \\
WSN & Yes & Yes & Peer-to-peer & 100 & Battery & $<3$ years & Small & IEEE 802.15.4 \\
RSN & Yes & Yes & Asymmetric & 3 & Harvested & Indefinite & Small & None \\
\hline
\end{tabular}

\section{Applications}

The Internet of Things with its special and unique characteristics has made changes for a better and easier lifestyle.There will be a wide range of applications and innovations among which only few have been discussed so far. The surrounding devices in house, travel, disease time, work, exercise, etc. now possess primitive intelligence with no connection with each other. The Internet of Things makes the nature of things smarter and create an expended network by connecting the objects.

The application areas of Internet of things are as follows:

A) Transportation and Logistics

B) E-health

c) Smart environment (house, work office, factory)

d) Personal and social areas

\section{Transportation and Logistics}

Advanced cars, trains, buses, and other means of transportation such as bicycles could be controlled on routes by sensors, actuators and processors. By equipping the roads and transport machineries with tags and sensors, these devices could be connected with each other by traffic signs. The communication and exchange of information between road transport and traffic signs which are the components of transportation help the improved management of traffic. You can also manage public transportation for 
better routing to destination. Managing for detection of goods in large warehouses, helping the tourism industry by providing detailed information of road status and tourist sites as well as monitoring the status of public transport facilities are amongst the other applications of Internet of Things in transport industry.

\section{Supporting}

Real-time processing technology established on the basis of RFID and NFC technologies could be very helpful in the realization of real-time monitoring on cases such as product supply chain from purchasing raw materials to shipping, storage, distribution and sales of production, sales returns process and aftersales services. The application of this technology also facilitates the procedure of receiving information about the products available in stores precisely and at different times. By this method, the institutions and companies and in fact the total supply chain could send the essential information to producing company in the least possible time. The chain stores and producing institutions which utilize the traditional method need 120 days since receiving customer information until they produce and send the products to store. However the modern stores (e.g. Wall Mart \& Metro) need just a few days by this technology. Actually they could receive the data by sensors in shopping centers within only a few days, immediately produce the products and deliver them to the stores across the country. It also leads to reduced warehousing costs and maintenance of instruments and raw materials. Besides the real-time access to ERP program could help the stores to offer more accurate and comprehensive information on products to customers.

\section{Driving}

The Cars, trains and buses moving on the road and on the track can provide useful information for better and safer travel for the driver and passengers Using sensors and tags that are installed on them. For example, we can point to Train Collision Avoidance System and Monitoring and Transporting System of Poisonous and Hazardous Material. The state organizations can do better planning for transportation management using very detailed information of the status of the roads and traffic. For example, with a road accident they can quickly take action to check the route and announce the alternative routes to drivers by radio in order to reduce the traffic load. The big companies such as international transportation companies could apply this technology to discover optimal routes and so save energy consumption. By combining information about cars, ships and planes that carry the load, more precise information could be offered to company and customer about delivery time, latency, etc. Moreover, with this information and receiving information about the status of warehouses products, we could take act for automated management of goods.

\section{Mobile Ticket}

Electronic information panels, labels and paper posters installed by passenger transport companies include information about the cost, schedule, departure and explanation of special services to customers. Using the panels and labels equipped with an NFC electronic tag, a small screen, keyboard and identification number, we could offer more information and services to customers. The customer can close his mobile phone to the device, receive the real-time information about origin, destination, cost, time, type of vehicle, number of passengers, the number of empty seats, special services, etc. and save them in his mobile phone. The customer can immediately use the keyboard and buy the ticket.

\section{Environmental Monitoring}

Perishable food such as fish, fruits, meat and dairy products are major and important parts of the food products. There are thousands of distance from production to distribution and delivery of these products to customers. Since they are perishable and sensitive products, environmental conditions such as temperature, humidity, pressure, etc. should be regularly measured and controlled during the transport of these goods to destination. Pervasive Processing Technology can be very useful in improving the quality of products in the supply chain.

\section{Tourism Industry}

Submit Date: 10.05.2016, Acceptance Date: 25.06.2016, DOI NO: 10.7456/1060JSE/041 Copyright $(\mathcal{C}$ The Turkish Online Journal of Design, Art and Communication 
The tourist sites could store exceeding information in the location by installing the NFC tags. Information about historical events, maps, translation phrases, etc. could be stored on the tag. The tourists and visitors could make their mobile phones close to these tags and so receive and save essential information in their phones. The communication between tags and their connection to central system could provide the ground for preparation of electronic map from tourist sites and attractions or even a certain location across the country.

\section{E-health}

E-health is one of the applicable and useful areas in Internet of Things. Applications of this group are divided into tracking (patients and staff), identification and authentication and automated data collection.

\section{Tracking}

The purpose of tracking is identifying and pursuing people or moving objects which encompass the precise tracking locations (tracking of patients to reach and better quality of medical services in hospitals), tracking people and objects passing certain places. With this technology, the exact location of people (patients and staff) and other objects used in the E-health environment could be identified.

\section{Patients Identification}

Identification and authentication of patients is primarily to prevent medical errors. It has been noticed that the injection by hospital staff or doctors has caused injuries to patients due to name similarity. This technology reduced the medical errors and damages caused by these errors to the patients by providing detailed information of the characteristics and history of disease, hospitalization and prescription records, comments and previous medical studies.

\section{Data Collection}

The purpose of automatic collection of data is reducing the time for data review, optimizing the automation process (data entry and error correction), automatic medical care and management of medical services. This technology requires communication between RFID equipment and other electronic clinic equipment.

\section{Senor and Tracking Patient Status}

The sensors make the implementation of centralized medical affairs on patients possible. The accurate diagnosis of the patient's condition and provision of information to the physician at proper time and based on the patient's health indices are amongst its uses. Performance of distant medical procedures, monitoring the patients' compliance with prescribed medications and patient care can be achieved with this technology.

\section{Smart Environments}

A smart environment is a place which takes the advantage of a kind of intelligence and make the working place very convenient for people in it. This environment could be a house, working office, place for rest and exercise or a factory.

\section{Comfortable House and Office}

The sensors and tags installed in working places and houses can make life much easier. For example, the heating systems could use the existing sensors to be adjusted to ambient temperature and provide optimum temperature. The lights and home lamps can adjust themselves (on or off) with regard to the ambient lighting conditions and the position of day and night. The interior devices will have the opportunity to be connected to a central warning system and be controlled by this system. For example, in the downtime or failure of one of the devices, an audible or electronic alert would be immediately produced by a warning system. Besides, the warning system could turn off the devises which are not are 
not used to save energy. This technology could change the houses and working office into comfortable places and save a great deal of energy.

\section{Industrial Plants}

Smart equipment has been widely used in various industries such as automotive, electronics, food industry. The smart equipment of industries could manage the more optimized automation processes of product. For example the tags could be installed on the pieces needed for production. So when the tag reaches its position, the robot can read the information stored in the chip (chip no. and other information) by RFID terminals installed on it. The robots can make decision about the piece and the type of operation that should be performed (e.g. welding). The robots can also manage the production process by reading the information on chips and recording their number. For example by operations on certain number of pieces, they lead the production process to the second phase (coloring).

\section{Smart Museums and Gyms}

Gyms and museums are interesting examples of taking advantage of the Internet of Things. In smart museums, certain conditions must be applied to represent various historical periods such as ancient Egypt or Ice Age so that the visitors could be intimately familiar with the atmosphere of that era. The responsibility of sensors or in fact The Internet of Things is transmission of data related to environmental conditions, heating and cooling systems, wind generation, etc. In this case the date of aforesaid periods could be simulated with physical conditions. This technology could also be applied in fitness centers and gyms. The coach can record the program of an athlete in a device (number of movements on different machines, time and pressure). After starting the activities of athlete, the sensors sent to the device activity information to device. The device on the other hand controls the manner of exercise movements by comparing the athlete activity and the data recorded by the coach so that it does not exceed the defined record.

\section{Personal and Social Areas}

Internet of Things in this area can play a role in communication between the people. In fact the Internet of Things creates interaction between persons, makes new social relations between human beings and improves them. In fact, with this technology, all events and things like traveling, meeting friends, going to football games, sleeping, traveling, etc. are automatically transferred as messages to others (friends) and others can easily be informed of the status of our life.

\section{Social Networks}

Automatic update of our social networking activities by social networks such as web portals, Twitter and Facebook are amongst the applications of this technology in the field of social networks. Tags and RFID technology can monitor our activities and immediately upload information in social networks. Therefore the events related to our physical environment are always real-time updated in the virtual environment.

\section{Historical Queries}

Historical queries about objects and events allow the user to discuss his activity trends over time. It can be useful for cases such as business and trade which occurs during long periods of time. After recording the events, the system could create a digital diaries of events and offer it to user. By studying past events and memories, the user can extract the required information and make decisions on the authenticity of activities, effectiveness of previous decisions, etc. which improve his activities.

\section{Object Detection and Thief Prevention}

As mentioned, in this technology, all surrounding objects are identifiable by RFID terminals through installing sensors and tags on them. For example a glass with tag could be identified by passing besides devices with the ability to read RFID. The RFID reader equipment, with the advent of this technology, will be mostly installed in places such as hotels, houses, fuel stations, stores, streets and roads. Now 
consider that the simplest application of this technology is an objects detection engine which is able to search for objects in the city or country. The object detection engine shows the last location where the object was detected using information collected from the city. It also can present a historical record of the places from which the object is passed.

Suppose that your watch with an internal tag is a stolen and the thief has transferred it from Hamadan to Tehran. From the moment that the watch was stolen and at the time it was being shipped, it has passed beside the existing RFID equipment in streets, bus way, etc. The watch information is being read from the tags installed in it and is provided to detection engine. In order to find your lost watch, it is necessary to enter the RFID tag number in detection engine so that it can show you the latest place where it has been.

\section{Confirm and Prevent Natural Disasters}

In recent years, the unexpected and natural disasters such as floods, earthquakes, mining loss and forest fires have repeatedly occurred. The Internet of Things can play a key role in timely announcing and preventing of this type of disasters. The timely notice of the occurrence of such cases is very important to avoid damage and casualties. As we know, accurate information of the time of such disasters requires physical presence in extremely dangerous conditions such as deep in earth or places or high temperature places. So the wireless sensor network (Internet of Things) can receive and process information and environmental conditions of high-risk locations where there is potential risk of natural disasters. This information could be disposed to the authorities so that they act to evacuate residents, organize resources to deal with the crisis and plan the situation carefully. Besides this technology could be useful to detect and help the lifeguard officers to help the workers and individuals trapped in disasters.

\section{Open Issues}

Although the technologies that realize the idea of the Internet of Things such as wireless sensors and RFID networks were studied in section 3, there is still a need for further researches in this area. In this section we first review the standards of various technologies associated with the Internet of Things. Then we will work on the very important issues that need to be discussed. In the first 5-1 sections, issues related to standards and networks should be examined. In section 5-2 the security issues and the security of payment data will be regarded. Section 5-3 discusses the issues on addressing and network.

Table (2): Comparison of Different Standards and their Specific Properties in Internet of Things

\begin{tabular}{|c|c|c|c|c|c|}
\hline Standard & Objective & Status & $\begin{array}{l}\text { Comm. } \\
\text { range }(\mathrm{m})\end{array}$ & $\begin{array}{l}\text { Data rate } \\
(\mathrm{kbps})\end{array}$ & $\begin{array}{l}\text { Unitary } \\
\text { cost }(\$)\end{array}$ \\
\hline \multicolumn{6}{|c|}{ Standardization activities discussed in this section } \\
\hline EPCglobal & $\begin{array}{l}\text { Integration of RFID technology into the electronic product code (EPC) framework, } \\
\text { which allows for sharing of information related to products }\end{array}$ & Advanced & $\sim 1$ & $\sim 10^{2}$ & $\sim 0.01$ \\
\hline GRIFS & $\begin{array}{l}\text { European Coordinated Action aimed at defining RFID standards supporting the } \\
\text { transition from localized RFID applications to the Internet of Things }\end{array}$ & Ongoing & $\sim 1$ & $\sim 10^{2}$ & $\sim 0.01$ \\
\hline $\mathrm{M} 2 \mathrm{M}$ & $\begin{array}{l}\text { Definition of cost-effective solutions for machine-to-machine (M2M) } \\
\text { communications, which should allow the related market to take off }\end{array}$ & Ongoing & N.S. & N.S. & N.S. \\
\hline 6LoWPAN & Integration of low-power IEEE 802.15.4 devices into IPv6 networks & Ongoing & $10-100$ & $\sim 10^{2}$ & $\sim 1$ \\
\hline ROLL & Definition of routing protocols for heterogeneous low-power and lossy networks & Ongoing & N.S. & N.S. & N.S. \\
\hline \multicolumn{6}{|c|}{ Other relevant standardization activities } \\
\hline NFC & Definition of a set of protocols for low range and bidirectional communications & Advanced & $\sim 10^{-2}$ & Up to 424 & $\sim 0.1$ \\
\hline $\begin{array}{l}\text { Wireless } \\
\text { Hart }\end{array}$ & $\begin{array}{l}\text { Definition of protocols for self-organizing, self-healing and mesh architectures over } \\
\text { IEEE 802.15.4 devices }\end{array}$ & Advanced & $10-100$ & $\sim 10^{2}$ & $\sim 1$ \\
\hline ZigBee & $\begin{array}{l}\text { Enabling reliable, cost-effective, low-power, wirelessly networked, monitoring and } \\
\text { control products }\end{array}$ & Advanced & $10-100$ & $\sim 10^{2}$ & $\sim 1$ \\
\hline
\end{tabular}

\section{Standardization}

A wide volume of researches have been conducted on standardization and implementation of Internet of Things by the scientific communities around the world. The most important of them include Auto-ID Laboratory which holds the results of previous researches, European Commission and European 
Standards Organizations (Such as ETSI, CEN, CENELEC, etc.) with the cooperation of international institutions (such as ISO, ITU) and other research institutions and centers (such as IETF, EPC Global). More information is obtained for further researches from European Telecommunications Standards Institute (ETS) and Internet Engineering Task Force (IETF). The 6LoWPAN is being designed with the purpose of IPv6 protocol optimization to work with devices with low energy consumption and data exchange rate. ROLL is also presented with the purpose of standardization and optimized protocols for IoT routing and related equipment [9] [10].

Table 2 demonstrates the most important features of present standard, their current situation, communication frequency range, data exchange rate and devices expenses.

\section{Security Issues}

People resist the Internet of Things because of the absence of public confidence. This lack of trust arises when people have no confidence that the where information collected by the Internet of Things is stored and who will access them. Further discussion on security issues on Internet of Things is to be continued in two parts of security and privacy.

\section{Security}

The Internet of Things is potentially vulnerable to attack. Firstly because if the objects are installed and left usually in one place for a long time, there is the possibility of being attacked and touched by hackers. Secondly because the wireless technology is usually applied, it is much easier to eavesdrop the information. Thirdly the limitation of objects in consuming energy and processing, many security plans could not be implemented in them.

\section{Privacy}

Privacy is rooted in human civilization and its protection and maintenance has been long considered. Privacy protection is also included in the laws of all countries. So these laws prevent the arrival of new technologies such as the Internet of Things into individuals' privacy.

\section{Network Addressing}

The Internet of Things include many nodes. The data provided by each node regardless of the status of user should have recovery access. This concept requires addressing the policies of network optimization. Now the Ipv4 uses a four part addressing for addressing to each node and so we know the number of addresses is running out. However we need a IP for each object to realize the Internet of Things. It is clear that IP version 4 cannot be used for this purpose. As we said, 6LoWPAN 0 uses version 6 for wireless communication devices with less load which is able to create $2^{128}$ number of separate address or in other words $3.4 * 10^{38}$ different IP. This number is $7.9 * 10^{28}$ times more than number of IP version 4 . With this number of IP produced in version 6 , everything could have a especial address.

It seems that with IPv6 we could allocate a 128 byte ID to every FRID equipment. But according to EPC Global Standard, the RFID equipment uses the 96-64 identification numbers. To solve this problem, certain solutions should be allocated to change the 128 byte addresses into 64-96 byte. Many researches have been recently presented to solve this problem. For example, the method suggests that we can use the first 64 byte of Ipv6 for detection of tag and the second part for network addressing and respective Gateway for connection to internet. In this method, the gateway will manage the messages produced by tags to send them to Internet and take them back. In this way the payload of sent packet from Ipv6 type contains sent information. When exiting the Gateway, its departure address will be the combination of Gateway and Tag address. Together with the 64 -byte tag address and 64-byte Gateway address, a valid 128-byte address equal to IP v6 is created which is sent to Internet. On back way the Gateway will manage this part by separation of these two parts. This method will only answer that addresses 64-byte identifier used to tag. If a 96 -byte address used which is valid by EPC Global, another method should be 
applied. Another method is proposed to solve the problem which uses a proper network element called agent. The task of this agent is transforming the ID address (regardless of its size) into a valid 64 byte address that is used as IPv6 as network address. This agent should always update the address mapping information of varying length to 64 byte addresses.

The FRID message along with its header capsuled in IPv6 packet is shown in Figure 6.

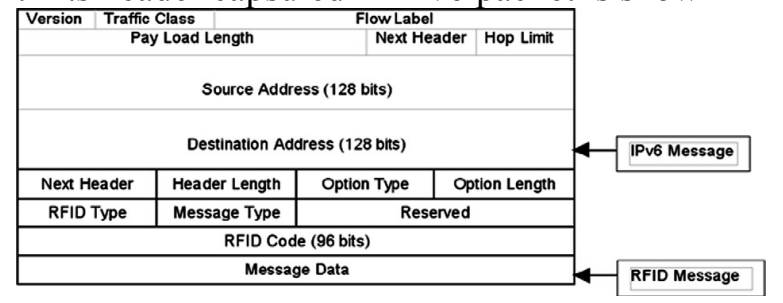

Figure 6: Capsulation of FRID message and header in IPv6 packet

The important note is that the mobility of objects is not regarded in aforesaid methods. In fact when an agent should be present on a Gateway which constantly updates the information or addresses of different lengths to be converted to 128 byte, it is essential to keep the respective device close to or connected to this agent or Gateway. This is despite the fact that in Internet of Things, the objects can move and shift from one place to another. To solve the problem of scalability, methods based on domestic factors (such as Mobile IP) could be applied instead of ways such as home location registers (HLR) and Visitor Location Register (VLR). In fact the Mobile-IP -based protocols do not apply a central server as a bottleneck for scalability problem. Another important issue is how to obtain the IP address of an object. In current Internet, the address of each host could be obtained by sending a request to the DNS server. DNS is responsible for changing the name of a host IP address. According to the concept of Internet of Things, the communications are mostly similar to the relation between two objects instead of two hosts. Therefore, we should create a new concept as ONS or service object name which returns the identifier address corresponding to the object tag with the name of an object. In fact, an ID tag will be converted to a URL by which the object information could be accessed. In this case, the ONS must work bidirectionally. It means that it could discover the ID profile and vice versa using RFID tag. Working bidirectional is not an easy task, so another service named object code conversion service (OCMS) is proposed. The features of OCMS were described in $* * * * * * * *$ by authors. According to the proposed model, the P2P communications were used for improvement of scalability. However the design and operational application of OCMS in widespread environment such as Internet of Things are also regarded by researchers.

The Internet of Things also requires a transport layer. In current Internet, the duty of transport layer is end-to-end congestion management. The Internet of things also uses the transport layer for establishment of a safe communication. The current transport layer is not able to response to the Internet of Things due to following reasons:

- Connection Setup: TCP is a connection set up and each session initiates with the process of communication using a Three-way Handshake Method which is an unnecessary operation for Internet of Things. Since the data exchange rate is very slightly in Internet of Things, the limited time for communication encompass major part of time required for being connected to Internet. Besides, the establishment of communication demands processing, sending and receiving certain information. This operation is also not essential due to the limitation of sources and energy consumption and should be avoided.

- Congestion Control: TCP is responsible for end-to-end congestion control. In Internet of things, the congestion control may lead to problems such as loss of yield in objects because the objects in Internet of Things uses wireless media. It is evident that wireless technology is a big challenge for environments using TCP. The congestion control is useless if the level of data transport is minor.

Submit Date: 10.05.2016, Acceptance Date: 25.06.2016, DOI NO: 10.7456/1060JSE/041 
It should be noticed that establishment of a TCP connection requires sending a message and receiving the result till the end of relation.

- Data buffering: TCP needs a buffer in sender and receiver for data storage. Actually the buffer is essential because the packets which are lost or did not reach the destination should be send again. The receiver buffer is needed for packet sorting and delivery by package in practical destination program. The management of these two buffers is very costly in both objects (in terms of energy consumption).

Therefore the TCP could not be used as a useful protocol for end-to-end data transport in Internet of things. No solution has been proposed for this problem up to now and further researches are required in this area. Besides, we still do not know how the exchange of information related to smart objects in the Internet of Things would be in future. This is a fundamental issue that must be determined. Then the research studies should be conducted on network infrastructures. So another important research topic is related to the traffic on these networks.

It is evident that the issue of traffic and its features in wireless networks are severely dependent on a practical program.it should be regarded that this issue is not a serious problem in wireless networks and occurs when a wireless node enters into public Internet. With regard to the properties of Internet of Things, this problem occurs when large number of data produced by sensor networks which are all sent to homogenous networks are delivered to Internet with diverse characteristics of traffic control.

Since the development of RFID-based systems in wide area and in distributed form is still at the beginning of a long path, the features of traffic is still not included in this study. In summary it can be said that traffic handled by the Internet of Things is still unknown to us. Internet service providers should also (if necessary) plan and develop their infrastructure for transporting the traffic of Internet of Things (This feature has no relation with traffic in wireless sensor networks).

Finally the designing of a traffic model and examining the needs to solve the traffic problems and creation of a proper solution (for all systems) and combining them provide the ground for better establishment of Qos. In fact, if the problem of Qos is solved in wireless sensor networks, the problems will be continued in RFID networks due to its especial features. So further studies should be done to support the quality of services in Internet of Things.

I believe that there are similarities between service quality of QoS in the Internet of Things and machine machine communication. So certain activities implemented on this are during the last years could be applied for Internet of things.

\section{CONCLUSION}

The Internet of things is rapidly expanding and will make a revolution in human life. The Internet of Things has great potentialities to create new dimension of lifestyle and could create a virtual life for human beings. The present paper indicates that to what extent the Internet of Things have impact on our life. We also tried to show that Internet of Things will be a dispensable part of our future life which bear technically different features compared to Internet. Besides, several parameters and features of present Internet such as congestion control etc. are obstacles for Internet of Things. The enabling technologies such as sensor networks, RFID-based systems and RFID sensor systems were also introduced in summary. The introduction and description of several prevalent standards created for realization of the idea of Internet of Things as well as the different applications of Internet of Things in different areas of transportation, supporting and smart environment were discussed. We also examined some noteworthy and open issues related to standardization, networking, addressing and security issues.

\section{REFERENCES}

LAN/MAN Standards Committee, IEEE Standard for Local and Metropolitan Networks, Part 15.4, LowRate Wireless Personal Area Networks(LR-WPANs), 2011.

Traub, Ken, The GS1 EPCGlobal Architecture Framework, 2013.

Submit Date: 10.05.2016, Acceptance Date: 25.06.2016, DOI NO: 10.7456/1060JSE/041

Copyright (C) The Turkish Online Journal of Design, Art and Communication 
UID Center, Ubiquitous ID Architecture, 2010.

Ashton, Kevin, Internet of Things, Procter \& Gamble (P\&G) Presentation, 1999.

S. De Deugd, R. Carroll, K. Kelly, B. Millett, J. Ricker, SODA: service oriented device architecture, IEEE Pervasive Computing 5 (3) (2006) 94-96.

D. Giusto, A. Iera, G. Morabito, L. Atzori (Eds.), The Internet of Things: A Survey, Science Direct, 2010. Yang Feng, De-li, Liang, Yi-Duo, School of Management Science and Engineering, Dalian University of Technology, China, A Survey of The Internet of Things, Atlantis Press, 2010.

J. Pasley, How BPEL and SOA are changing web services development, IEEE Internet Computing 9 (3) 60-67, 2005.

Hui, Jonathan, W. Arch Rock, Culler, David, E., University of California, Berkeley, "Extending IP to Low-Power, Wireless Personal Area Networks", IEEE, 2008.

Ko, GeongGil, Terzis, Andreas, Johns Hopkins University, Connecting Low-Power and Lossy-Networks to the Internet, IEEE, 2011.

L. Srivastava, Pervasive, ambient, ubiquitous: the magic of radio, in: European Commission Conference "From RFID to the Internet of Things", Bruxelles, Belgium, March 2006.

National Intelligence Council, Disruptive Civil Technologies - Six Technologies with Potential Impacts on US Interests Out to 2025 - Conference Report CR 2008-07, April 2008, http://www.dni.gov/nic/NIC home.html 